\title{
ASSOCIATIONS BETWEEN PROLONGED GRIEF DISORDER, DEPRESSION, POSTTRAUMATIC STRESS DISORDER, AND ANXIETY IN RWANDAN GENOCIDE SURVIVORS
}

\author{
SUSANNE SCHAAL \\ Department of Psychology, University of Konstanz, Konstanz, Germany \\ JEAN PIERRE DUSINGIZEMUNGU \\ Department of Psychology, University of Butare, Butare, Rwanda
}

\author{
NADJA JACOB
}

Department of Psychology, University of Konstanz, Konstanz, Germany

FRANK NEUNER

Department of Psychology, University of Bielefeld, Bielefeld, Germany

\section{THOMAS ELBERT}

Department of Psychology, University of Konstanz, Konstanz, Germany

A number of studies have demonstrated that symptoms of prolonged grief disorder (PGD) represent a symptom cluster distinct from bereavement-related depression, anxiety, and posttraumatic stress disorder (PTSD). The aim of the present study was to confirm and extend these findings using the most recent criteria defining $P G D$. The authors interviewed a total of 400 orphaned or widowed survivors of the Rwandan genocide. The syndromes were strongly linked to each other with a high comorbidity. Principal axis factoring resulted in the emergence of 4 different factors. The symptoms of depression, along with the cognitive, emotional, and behavioral symptoms of $P G D$, loaded on the first factor, symptoms of anxiety on the second factor, symptoms of PTSD on the third factor, and the separation distress symptoms of PGD on the fourth factor. This indicates that the concept of

We would like to thank the psychologists and psychology students from the National University of Rwanda who assisted us with data collection. We deeply appreciate the great enthusiasm, trust, and openness of the orphans and widows and the many others who have supported our work. Research was funded by the Ministry of Science, Research and the Arts of Baden Württemberg and by the Deutsche Forschungsgemeinschaft.

Address correspondence to Susanne Schaal, PhD, University of Konstanz, Department of Psychology, Universitätsstrasse 10, 78457 Konstanz, Germany. E mail: Susanne.Schaal@ uni konstanz.de 
PGD includes symptoms that are conceptually related to depression. However, the symptom cluster of separation distress presents a grief-specific dimension that may surface unrelated to depressive symptoms.

Following the death of a significant other, most bereaved individuals accommodate loss and return to preloss levels of functioning relatively soon after the bereavement. However, a minority of people fails to return to normal functioning and may develop chronic grief reactions, referred to as prolonged grief disorder (PGD). The concept of PGD has only recently emerged in the contemporary literature; Prigerson and coworkers developed and empirically tested consensus and diagnostic criteria for this disorder (Prigerson et al., 2009; Prigerson, Vanderwerker, \& Maciejewski, 2008). However, bereaved persons often present with symptoms of depression (Harrison \& Harrington, 2001; Vinkers, Gussekloo, Stek, Westendorp, \& van der Mast, 2004), posttraumatic stress disorder (PTSD; Kersting et al., 2007; Rynearson \& McCreery, 1993; Schut, De Keijser, Van Den Bout, \& Dijkhuis, 1991), or anxiety (Kersting et al., 2007; Neria et al., 2007), and, therefore, the question of how to cluster the set of symptoms has remained a challenge for revising diagnostic categories.

The present study examined associations between PGD, depression, PTSD, and anxiety in a sample of orphaned and widowed survivors of the Rwandan genocide, that is, in survivors that had all experienced (a) traumatic events, which constitutes a precondition for a diagnosis of PTSD, and (b) the loss of a significant other, which is a precondition for PGD. Depression may develop as a consequence of both.

In 1994, Rwandans experienced one of the worst genocides of the 20th century. Over the course of 3 months between 800,000 and 1,000,000 Rwandese were killed. The fighting between two artificially divided "ethnic" groups of the Hutu majority and the Tutsi minority was executed with a brutality beyond imagination. Neighbors were killing neighbors, children murdered children, teachers were killing their students, and husbands were brought to murder their wives. The aim was the extermination of the Tutsi minority and the Hutu opponents. As a result of the genocide, the majority $(70.9 \%)$ reported having lost a close family member during the fighting (Pham, Weinstein, \& Longman, 
2004). During the Rwandan genocide, survivors had to endure a wide range of traumatic events including violent losses (Dyregrov, Gupta, Gjestad, \& Mukanoheli, 2000; Schaal \& Elbert, 2006). It was common for many genocide survivors to have witnessed the violent death of family members while experiencing lifethreatening conditions themselves. As a result of such traumatic losses, symptoms of PGD and PTSD may or may not co-occur. Such an interplay between trauma and loss and their outcomes has rarely been studied. Green (2000) and Green and colleagues (2001) argued that the violent loss of a person should be viewed as a traumatic stressor and therefore the psychological reactions that occur after bereavement display a great overlap with symptoms of PTSD. Given that stressors need to elicit a strong physiological response, usually an alarm reaction, in order to qualify as traumatic, such a view seems questionable. It is therefore not surprising that symptoms of PTSD fail to adequately capture the distress of those who suffer from PGD as a result of violent loss of a significant other, as reported by Neria and Litz (2004). These authors argue that the "loss as trauma" framework (e.g., Green, 2000) would not sufficiently acknowledge the unique reactions following bereavement.

Then again, it is plausible to assume that symptoms of PTSD after a violent loss can interfere with the grieving process. Persisting anxiety supports avoidance behavior, which interferes with processing grief-related information; that is, the work of mourning will remain incomplete and eventually grief reactions may become aggravated. Hence, some authors have suggested that the trauma must be addressed before grief can be targeted (Lindy, Green, Grace, \& Titchener, 1983; Pynoos \& Nader, 1988). Similarly, Eth and Pynoss (1994) demonstrated that children who witnessed the murder of their parents displayed posttraumatic stress responses that interfered with their ability to successfully grieve.

A number of studies have documented a substantial association between PGD and PTSD (Melhem, Moritz, Walker, Shear, \& Brent, 2007; Pfefferbaum et al., 2001) and between PGD and depression (Kim \& Jacobs, 1991; Melhem et al., 2007; Momartin, Silove, Manicavasagar, \& Steel, 2004). Moderate to high correlations between PGD and depression and between PGD and PTSD have been found (Boelen \& Prigerson, 2007; Boelen \& van den Bout, 2005; Bonanno et al., 2007; Hogan, Worden, \& Schmidt, 


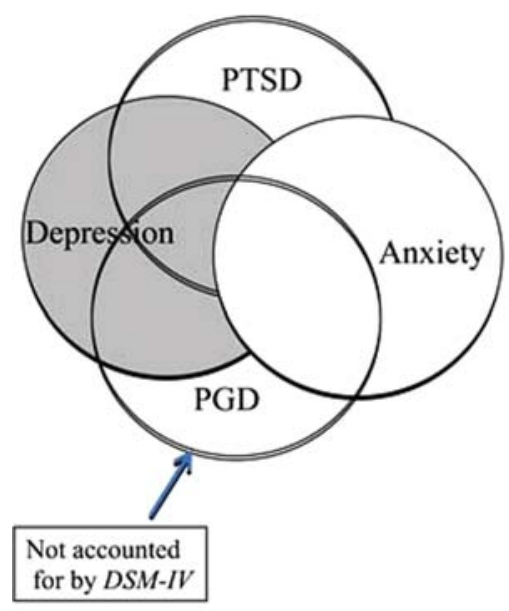

(a)

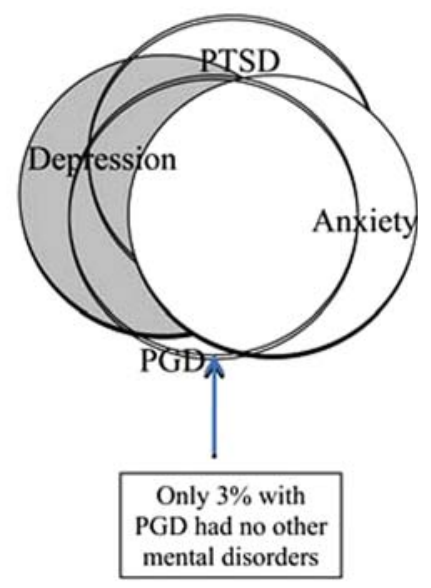

(b)

FIGURE 1 Schematic illustration of overlap between diagnostic symptom clusters derived from (a) the literature and (b) the present study: depression, posttraumatic stress disorder (PTSD), anxiety, and prolonged grief disorder (PGD; color figure available online).

2004; Melhem et al., 2004, 2007). The reported correlations between PGD and anxiety symptoms are low to moderate (Boelen \& Prigerson, 2007; Boelen, \& van den Bout, 2005; Melhem et al., 2007; Prigerson et al., 1996). Although there are substantial associations between these pathological constructs, diagnoses for PGD and major depressive episode (MDE) and PGD and PTSD have been shown to have a moderately high, but far from complete overlap with each other: The majority of persons with PGD or subsyndromal PGD did not meet the criteria for major depression (Horowitz et al., 1997; Melhem et al., 2004; Neria et al., 2007; Simon et al., 2007), for PTSD (Silverman et al., 2000; Simon et al., 2007), or for an anxiety disorder (Neria et al., 2007; Simon et al., 2007). More than one third of the participants who met the criteria for PGD did not meet the criteria for either MDE or PTSD (Bonanno et al., 2007; Neria et al., 2007; Silverman et al., 2000). Figure 1a presents a schematic drawing of the comorbid overlap. These results suggest that many people who present with significant and prolonged suffering are not accounted for by the Diagnostic and Statistical Manual of Mental Disorders (DSM; Figure 1a). Hence, in the last decade there has been a debate about 
whether PGD should be considered a separate diagnostic entity in the next edition of the DSM. This would be justified if it can be distinguished from other mental disorders like major depression, PTSD, and anxiety disorder. Several researchers have suggested that the symptoms of PGD result from a distinct nosological syndrome distinguishable from other DSM disorders. In a series of factor analytic investigations researchers were able to differentiate a grief-specific symptom factor from bereavement-related depression and anxiety (Boelen \& Prigerson, 2007; Boelen, \& van den Bout, 2005; Boelen, van den Bout, \& de Keijser, 2003; Chen et al., 1999; Dillen, Fontaine, \& Verhofstadt-Denève, 2009) or PTSD (Pivar \& Field, 2004). There is evidence that this cluster of symptoms is associated with functional impairments and substantial morbidity even after controlling for current psychopathology such as depression, PTSD and anxiety (Bonanno et al., 2007; Melhem et al., 2007; Simon et al., 2007). Furthermore, it seems that this set of symptoms - unlike the set used to diagnose depression - cannot be ameliorated by interpersonal psychotherapy, either alone or in combination with tricyclic antidepressant (Pasternak et al., 1991; Reynolds et al., 1999).

Within the presented framework, the aim of the present study was twofold. First, we wanted to examine PGD and its relationship to symptoms of depression, PTSD, and anxiety and to report on comorbidity among participants who met PGD criteria in a sample of orphaned and widowed survivors of the Rwandan genocide. To further understand the nature of the clinical response to both experiences, exposure to traumatic stress and loss of a loved one, we secondly wanted to investigate to what extent symptoms of PGD are distinct from symptoms of depression, PTSD, and anxiety. Even though we expected associations between these constructs and high rates of psychiatric comorbidity, we expected that PGD would appear as a syndrome distinct from bereavement-related symptoms of depression, PTSD, and anxiety (Figure 1a).

\section{Method}

\section{Procedure}

The study was conducted in Butare, Rwanda, in August 2007. It was approved by Rwanda's National Institute of Statistics, Kigali, 
and by the University of Konstanz Ethical Review Board. Eligible subjects were widows (female gender) and orphans (female and male gender) who were at least 18 years old at the time of the interview and who had experienced the Rwandan genocide in 1994. We chose widows for participation as the target of the genocide mainly was the extermination of male gender leaving numerous widows behind. We interviewed orphans and widows to ensure at least one loss experience. However, all but one person had faced multiple losses including the loss of a partner $(48.5 \%)$, the mother $(72.9 \%)$, the father $(90.7 \%)$, a sibling $(86.0 \%)$, a child $(38.8 \%)$, another family member $(96.8 \%)$, or another close person $(79.5 \%)$ with a total mean of five different types of experienced losses (Schaal, Jacob, Dusingizemungu, \& Elbert, 2010). All participants were fully informed of the study procedure and aims, and written informed consent was obtained from all subjects. Diagnostic interviews were carried out by 15 master's level psychologists and psychology students (seven female and eight male) from the National University of Butare, Rwanda. The various questionnaires were translated into Kinyarwanda and back-translated by master level psychology students from the University of Butare. Interviewers participated in an intensive 2-week training program led by two female psychologists (Susanne Schaal and Nadja Jacob). The training covered basic theoretical concepts as well as sensitive and empathic interviewing techniques. Acute suicidal participants received a brief counseling intervention after the end of the interview, and it was ensured that emotional arousal had diminished in all participants. Participants who were severely traumatized were offered therapy as part of a treatment trial.

The first interviews in the field were conducted under the supervision of the psychologists, and the interviewers received extensive feedback. Interviews were carried out in five of the following randomly selected sectors of Butare: Tumba, Mukura, Mbazi, Huye, and Ngoma. Three to five trained raters were randomly assigned to each sector, and in each sector three quarters were randomly selected (one quarter per person). Meetings were arranged every other day to supervise the quality of the interviews, to review the questionnaires, and to provide feedback. The study was conceived as a community-based study with a house-to-house survey. Interviewers went house-to-house, starting at a convenient location within the assigned quarter. Each subsequent house was 
approached until the required number of interviews was achieved. Dwellers were asked if any widows or orphans resided within the home. If an orphan or widow was identified by the family, the interviewer then clarified if inclusion criteria were met. Houses were reapproached at a later time if nobody was encountered or available at the first visit. If there were both a widow and an orphan living in the same household, both were interviewed, if available and willing. If more than one orphan was living in a household, one was chosen randomly for participation. The interview lasted about $2 \mathrm{hr}$ and was conducted in the respondent's home. After the interview, interviewees received 1,000 Rwandan francs (about 1.30 euros) for their participation.

\section{Instruments}

Sociodemographic information collected included gender, age, educational background, and religious affiliation. PGD was assessed using the Prolonged Grief Disorder Questionnaire (PG-13; Prigerson et al., 2008, 2009). The PG-13 is a structured diagnostic interview designed to assess a wide variety of potential PGD symptoms in the past month, using a 5-point scale (from 1 to 5) representing increasing levels of symptom severity. A PGD diagnosis requires that one of the proposed two "separation distress" symptoms (e.g., yearning, intrusive thoughts, intense pangs of separation distress) and five of the following nine proposed "cognitive, emotional and behavioral" symptoms receive a score of at least 4 (at least once a day or marked): feeling emotionally numb, feeling stunned or shocked, feeling that life is meaningless, confusion about one's role in life or diminished sense of self, mistrust of others, difficulty accepting the loss, avoidance of the reality of the loss, bitterness over the loss, and difficulty moving on with life. Symptoms must be present for at least 6 months and must be associated with significant functional impairment in social, occupational, or other important areas. The grief score includes the sum of the score of each of the 11 grief symptoms with a range of 11 to 55 . The PG-13 covers all symptoms that have recently been proposed for inclusion in DSM V and that have been described above (Prigerson et al., 2008, 2009). The questions of the PG-13 referred to the loss, which was personally experienced as the most disturbing (PGD-related loss). PTSD was assessed using the PTSD 
Symptom Scale-Interview (Foa \& Tolin, 2000). The PTSD Symptom Scale-Interview assesses the 17 DSM IV symptom criteria for PTSD and refers to symptoms experienced in the previous month. Each of the items was answered on a 4-point scale ranging from 0 (not at all/only one time) to 3 (5 or more times per week/almost always). A PTSD severity-score (possible scores range from 0 to 51) was computed by summing all symptom scores. Symptoms of anxiety and depression were measured using the Hopkins Symptom Checklist25 (Derogatis, Lipman, Rickels, Uhlenhut, \& Covi, 1974; Lee, Kaaya, Mbwambo, Smith-Fawzi, \& Leshabari, 2008). Each of the 10 and 15 items measuring anxiety and depression are scored on a 4-point response scale ranging from 1 (not at all bothered) to 4 (extremely bothered). Possible scores for the anxiety score range from 10 to 40 , for the depression score from 15 to 60. Following recommendations in the literature, a mean score of 1.75 has been defined as the cutoff point for depression and anxiety symptoms (Mollica, Wyshak, de Marneffe, Khuon, \& Lavelle, 1987; Winokur, Winokur, Rickels, \& Cox, 1984). All diagnostic instruments (including the self-rating scales) were administered as clinical interviews.

\section{Participants}

In the present study, 400 widows and orphans were interviewed (widows: $n=194,48.5 \%$; orphans: $n=206,51.5 \%$ ). Eighteen subjects who were approached rejected participation in the trial and three subjects did not finish the interview. The sample consisted of 351 women $(87.7 \%)$ and 49 men $(12.3 \%)$. The participants' mean age was 37.18 years $(S D=16.73$, range $=18-97$ years). Further characteristics of the sample can be drawn from Table 1. For the majority $(62.0 \%, n=246)$, the PGD-related loss occurred during the genocide. A total of $8.0 \%(n=32)$ of the sample met criteria for probable PGD (Schaal et al., 2010). Participants were on average 25.72 years old $(S D=16.52$, range $=2-85)$ when they experienced the PGD-related loss and 11.5 years $(S D=4.15$, range $=1-38)$ had passed since the loss.

\section{Statistical Analyses}

Descriptive data are presented as frequencies (\%), mean scores, and standard deviations. Correlations (Phi coefficients, Pearson 
TABLE 1 Characteristics of the Sample $(N=400)$

\begin{tabular}{|c|c|c|c|c|c|}
\hline Characteristic & $\%$ & $n$ & $M$ & $S D$ & Range \\
\hline \multicolumn{6}{|l|}{ Sort of bereavement } \\
\hline Widow & 48.5 & 194 & & & \\
\hline Orphans & 51.5 & 206 & & & \\
\hline \multicolumn{6}{|l|}{ Sex } \\
\hline Male & 12.3 & 49 & & & \\
\hline Female & 87.7 & 351 & & & \\
\hline \multicolumn{6}{|l|}{ Having a partner } \\
\hline Yes & 30.0 & 120 & & & \\
\hline No & 70.0 & 280 & & & \\
\hline Age & & & 37.18 & 16.73 & 1897 \\
\hline Monthly income in Rwd francs ${ }^{a}$ & & & $6,845.81$ & $12,108.93$ & 0100,000 \\
\hline Completed school years & & & 4.93 & 3.50 & $\begin{array}{ll}0 & 18\end{array}$ \\
\hline \multicolumn{6}{|l|}{ Highest school degree obtained } \\
\hline No degree & 52.5 & 210 & & & \\
\hline Primary school & 37.0 & 148 & & & \\
\hline Secondary school & 4.8 & 19 & & & \\
\hline Apprenticeship & 5.5 & 22 & & & \\
\hline University & 0.3 & 1 & & & \\
\hline \multicolumn{6}{|l|}{ Religion } \\
\hline Catholic & 61.0 & 244 & & & \\
\hline Protestant & 23.3 & 93 & & & \\
\hline Islamic & 4.0 & 16 & & & \\
\hline Adventiste & 2.0 & 8 & & & \\
\hline Other & 6.0 & 24 & & & \\
\hline No religion & 3.8 & 15 & & & \\
\hline \multicolumn{6}{|l|}{ PGD related loss } \\
\hline Partner & 27.8 & 111 & & & \\
\hline Mother & 23.0 & 92 & & & \\
\hline Father & 21.5 & 86 & & & \\
\hline Child & 9.6 & 39 & & & \\
\hline Sibling & 11.8 & 47 & & & \\
\hline Another family member & 5.3 & 21 & & & \\
\hline Another person & 1.0 & 4 & & & \\
\hline
\end{tabular}

${ }^{a} 1,000$ Rwandan Franc 1.30 Euro.

correlation coefficients) were used to determine the relationships between psychiatric diagnoses and symptom scores. Betweengroup differences were analyzed using independent samples $t$ tests and nonparametric tests (Mann-Whitney $U$ test) if the precondition of normal distribution (Kolmogorov-Smirnov test) was not met. To examine the distinctness of PGD from depression, PTSD, and 
anxiety, a principal axis factoring with varimax rotation was carried out. We selected those six items from the PG-13, the PTSD Symptom Scale-Interview, and the Hopkins Symptom Checklist-25 that were among the most highly correlated with each scale's total score. All of the items were selected a priori to represent symptoms of PGD, depression, PTSD, and anxiety. Eigenvalues of 1 or greater were used to determine the number of factors emerging from the selected pool of items. Analyses were carried out using SPSS 16.

\section{Results}

\section{Associations Between PGD and Related Features}

Positive associations were found between the different diagnoses/ established cutoff points and scores. Moderate correlations were present between the PGD score and the depression score (Pearson $r=.63, p<.001)$, between the PGD score and the PTSD score (Pearson $r=.72, p<.001$ ), and between the PGD score and the anxiety score (Pearson $r=.60, p<.001$ ). PGD diagnosis was positively correlated with PTSD diagnosis $(\mathrm{Phi}=.31, p<.001)$, symptom criteria for depression $(\mathrm{Phi}=.29, p<.001)$, and anxiety $(\mathrm{Phi}=.22, p<.001)$.

As can be seen in Table 2, participants who fulfilled the criteria for PGD diagnosis reported a higher depression score, PTSD score, and anxiety score than those who did not fulfill criteria for the proposed PGD diagnosis criteria. Inversely, participants who met criteria for depression, PTSD, and anxiety had a higher PGD score compared to participants who did not meet the respective diagnostic criteria.

\section{Comorbidity of PGD}

The majority of participants who met the diagnostic criteria for PGD fulfilled comorbid symptom criteria for depression $(90.3 \%$, $n=28)$, a PTSD diagnosis $(84.4 \%, n=27)$, or symptom criteria for anxiety $(87.5 \%, n=28)$. The majority of the participants who met the diagnostic criteria for PGD $(71.9 \%, n=23)$ also met the criteria for all three related conditions (PTSD, symptom criteria for depression, and anxiety). A large percentage of the participants $(90.3 \%, n=28)$ who met the criteria for PGD simultaneously fulfilled at least two other comorbid disorders (symptom criteria for 


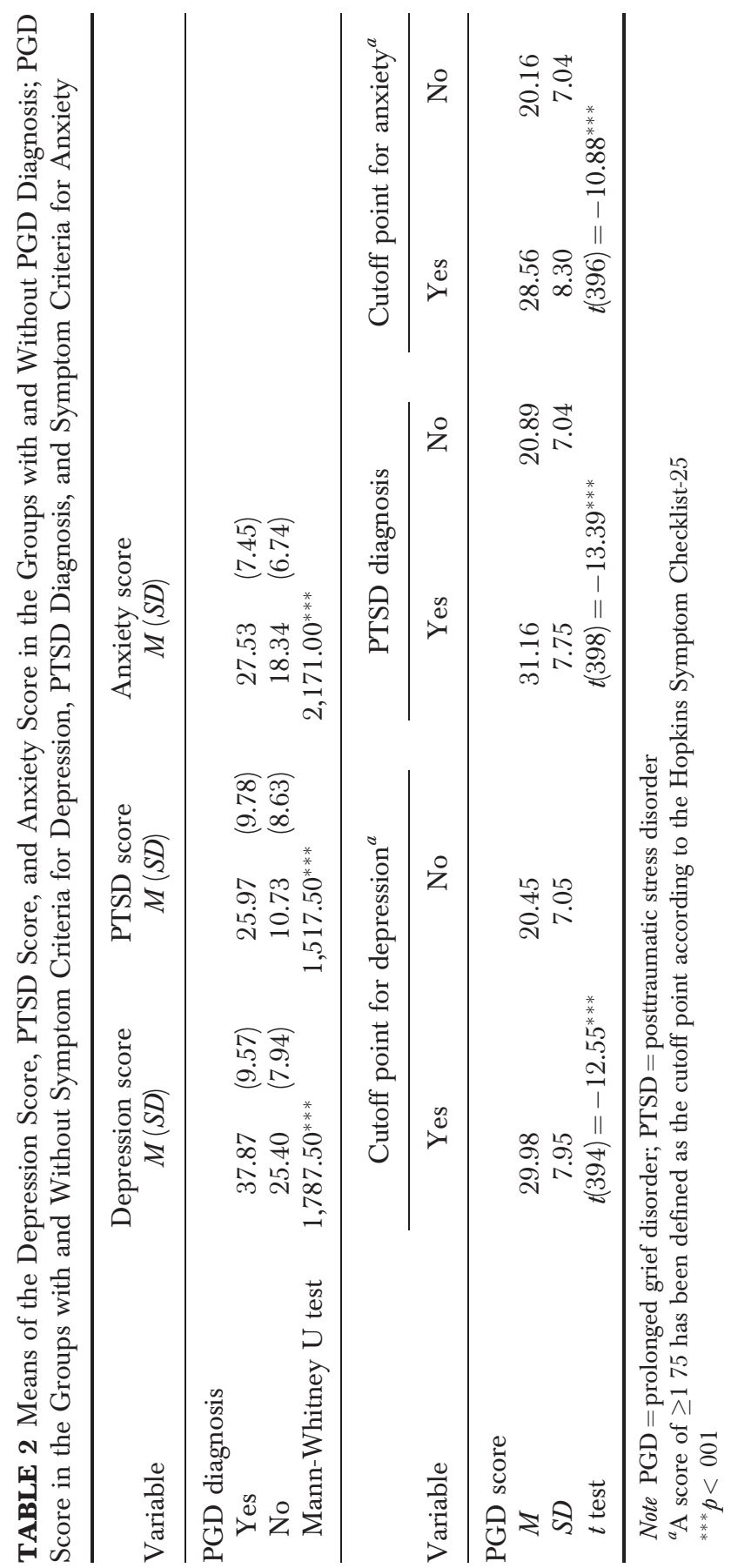


depression, symptom criteria for anxiety, or PTSD). Almost all participants $(96.9 \%, n=31)$ with PGD were diagnosed with at least one other mental disorder. Figure $1 \mathrm{~b}$ indicates these results schematically.

\section{Principal Axis Factoring}

The items clustered together in four different factors with eigenvalues greater than 1.0 accounting for $55.04 \%$ of the total variance. The cluster of symptoms loading on the different factors reflect depression (Factor 1), anxiety (Factor 2), PTSD (Factor 3), and PGD (Factor 4) and accounted for 19.29\%, 13.90\%, 12.36\%, and $9.49 \%$ of the variance, respectively. The symptoms of depression (e.g., feeling hopeless, feeling no interest in things) and the emotional, cognitive, behavioral symptoms of PGD (e.g., feeling life is unfulfilling, emotional numbness since the loss) loaded highly on the first factor (with factor loadings ranging from 0.55 to 0.75 ). The symptoms of anxiety (e.g., feeling fearful, heart pounding, or racing) loaded well on the second factor (with factor loadings ranging from 0.51 to 0.77), the symptoms of PTSD (e.g., unwanted memories, bad dreams) loaded on the third factor (with factor loadings ranging from 0.48 to 0.74 ), and symptoms of the Separation Distress subscale of the PG-13 formed the fourth factor (with factor loadings ranging from 0.57 to 0.83 ).

In general, the symptoms could be attributed to just one factor. However, it was still possible for a particular symptom to distribute its variance on two factors. The anxiety items "faintness or dizziness or weakness," "nervousness or shakiness," and "feeling tense or keyed up" loaded on both the anxiety and depression factors ( 0.51 vs. $0.42,0.54$ vs. $0.49,0.45$ vs. 0.52 , respectively). This suggests that these symptoms may be important elements of either disorder. The PTSD item "foreshortened future" loaded on the PTSD factor and the depression factor (0.54 vs. 0.50), and the PTSD item "hypervigilence" loaded on the PTSD and the anxiety factor (0.47 vs. 0.53 ). We acknowledge that these symptoms may actually have served to define both of these factors. The PG-13 item "confusion about one's role in life or diminished sense of self" of the Cognitive, Emotional, and Behavioral subscale loaded low on all four factors with factor loadings of 0.41 for depression, 0.39 for PTSD, 0.37 for PGD, and 0.14 for anxiety. Table 3 presents the items loading on the different factors. 
TABLE 3 Factor Loadings for Symptoms of Depression, PGD, PTSD, and Anxiety

\begin{tabular}{|c|c|c|c|c|}
\hline Symptoms & $\begin{array}{l}\text { Loading on } \\
\text { Factor } 1 \\
\text { (Depression) }\end{array}$ & $\begin{array}{c}\text { Loading on } \\
\text { Factor } 2 \\
\text { (Anxiety) }\end{array}$ & $\begin{array}{c}\text { Loading on } \\
\text { Factor } 3 \\
\text { (PTSD) }\end{array}$ & $\begin{array}{c}\text { Loading on } \\
\text { Factor } 4 \\
\text { (PGD) }\end{array}$ \\
\hline \multicolumn{5}{|l|}{ Symptoms of depression } \\
\hline Feeling hopeless & 0.65 & 0.04 & 0.34 & 0.05 \\
\hline Feeling blue & 0.55 & 0.41 & 0.01 & 0.16 \\
\hline Feeling lonely & 0.56 & 0.24 & 0.22 & 0.09 \\
\hline Feeling trapped or caught & 0.68 & 0.18 & 0.02 & 0.10 \\
\hline Feeling no interest in things & 0.75 & 0.04 & 0.13 & 0.13 \\
\hline Feeling worthless & 0.69 & 0.17 & 0.19 & 0.03 \\
\hline \multicolumn{5}{|l|}{ Symptoms of PGD } \\
\hline Feelings of yearning & 0.15 & 0.10 & 0.11 & 0.83 \\
\hline Emotional pain & 0.17 & 0.15 & 0.18 & 0.82 \\
\hline Stunned or shocked & 0.13 & 0.16 & 0.40 & 0.57 \\
\hline Confusion about role & 0.41 & 0.14 & 0.39 & 0.37 \\
\hline Emotional numbness & 0.48 & 0.01 & 0.31 & 0.21 \\
\hline Feeling life is unfulfilling & 0.62 & 0.15 & 0.31 & 0.19 \\
\hline \multicolumn{5}{|l|}{ Symptoms of PTSD } \\
\hline Unwanted memories & 0.22 & 0.13 & 0.64 & 0.31 \\
\hline Bad dreams/nightmares & 0.16 & 0.17 & 0.74 & 0.11 \\
\hline Physical reaction & 0.15 & 0.33 & 0.60 & 0.14 \\
\hline Isolation & 0.36 & 0.24 & 0.48 & 0.23 \\
\hline Foreshortened future & 0.50 & 0.12 & 0.54 & 0.09 \\
\hline Hypervigilence & 0.09 & 0.53 & 0.47 & 0.06 \\
\hline \multicolumn{5}{|l|}{ Symptoms of anxiety } \\
\hline Suddenly scared for no reason & 0.06 & 0.72 & 0.23 & 0.14 \\
\hline Feeling fearful & 0.09 & 0.77 & 0.20 & 0.09 \\
\hline Faintness/Dizziness/Weakness & 0.42 & 0.51 & 0.21 & 0.07 \\
\hline Nervousness/Shakiness inside & 0.49 & 0.54 & 0.08 & 0.19 \\
\hline Heart pounding or racing & 0.23 & 0.76 & 0.09 & 0.07 \\
\hline Feeling tense or keyed up & 0.52 & 0.45 & 0.05 & 0.18 \\
\hline
\end{tabular}

Note. PGD prolonged grief disorder; PTSD posttraumatic stress disorder. Eigenvalue after rotation 4.63, variance explained 19.29; eigenvalue after rotation 3.34, variance explained 13.90; eigenvalue after rotation 2.97, variance explained 12.36; eigenvalue after rotation 2.28, variance explained 9.49. Bold highest factor loading of the item.

\section{Discussion}

As a first goal, we investigated the associations between the symptom criteria set for PGD proposed by Prigerson and colleagues (2008) and depression, PTSD, and anxiety in a sample of Rwandan orphans and widows who experienced the genocide in 1994 and analyzed comorbidity among participants who met PGD criteria. 
Secondly, we examined whether PGD constitutes a syndrome that may appear independently from symptoms of depression, PTSD, and anxiety when both traumatic stress and loss of a loved one have been experienced. We found that PGD was strongly associated with depression, PTSD, and anxiety (Figure 1b). Whereas the symptom clusters of depression, PTSD, and anxiety represented distinct factors, the symptom cluster of PGD was separated into two factors, one of them reflecting depression.

We also found substantial relations between symptoms of PGD, depression, PTSD, and anxiety. This result is comparable with a number of studies that have reported moderate to strong associations between PGD and depression, between PGD and PTSD, and between PGD and anxiety (e.g., Melhem et al., 2007). A considerable overlap between the disorders was found: Symptoms of depression, PTSD, and anxiety were more common among widows and orphans with PGD compared with those who did not fulfill the proposed diagnostic criteria. Inversely, survivors who met criteria for PTSD and symptom criteria for depression or anxiety reported more PGD symptoms than those who did not meet the respective diagnostic criteria.

Many researchers have reported that PGD diagnosis only partially overlaps with the diagnosis for depression, PTSD, or anxiety disorder (Neria et al., 2007; Simon et al., 2007). In our sample, a high rate of psychiatric comorbidity was found. Almost all widows and orphans with PGD diagnosis (97\%) fulfilled criteria for at least one other associated condition (either diagnostic criteria for PTSD and symptom criteria for depression or for anxiety). Moreover, the majority of participants $(72 \%)$ meeting diagnostic criteria for PGD simultaneously received a diagnosis for PTSD and fulfilled the symptom criteria for depression and anxiety. The high psychiatric comorbidity found in our sample shows that massive stress may have long-lasting effects. This underlines the importance to assess for multiple comorbid conditions even many years after trauma confrontation and loss experiences. A recent study demonstrated that symptoms of PTSD and depression can be treated effectively in this context (Schaal, Elbert, \& Neuner, 2009a). The documented comorbidity in our study is stronger than the rates reported in other studies and questions the usefulness of a categorical model for mental disorders (Figure 1a vs. Figure 1b). According to Bonanno and coworkers (2007) and Neria and colleagues (2007) 
only half of the participants $(45 \%$ and $51 \%)$ who met PGD criteria simultaneously met criteria for either major depressive disorder or PTSD.

Loss by traumatic means can lead to prolonged grief reactions (Melhem et al., 2004), depression (Melhem et al., 2004), symptoms of anxiety (Neria et al., 2007), or PTSD (Rynearson \& McCreery, 1993) and therefore constitutes a risk factor for all measures of psychopathology used in our sample. All but one participant had experienced multiple losses, and most of the participants in the present study $(62 \%)$ experienced their worst loss during the genocide, indicating that trauma exposure and bereavement coincided in the majority of participants. The violent nature of the loss was associated with more severe grief reactions (Schaal et al., 2010). It is likely that the high incidence of traumatic loss in our sample contributed to the high diagnostic overlap compared to other studies. Because our data are cross sectional and correlational in nature, we cannot determine what syndrome predicted what disorder. On the one hand, it is possible that the loss of a loved one may precipitate the onset of some related features as depression, PTSD, and anxiety. The results of Mehlem and colleagues (2004) give evidence that PGD predicts the onset or course of depression and PTSD, even after controlling for depression. On the other hand, it is possible that a preexisting mental disorder may facilitate the development of PGD, as suggested by Simon and coworkers (2007). It is also likely that PTSD symptoms might interfere with the survivors' ability to successfully complete the mourning process. Any thoughts about the deceased might automatically trigger trauma reminders and lead to associated fear and PTSD symptoms. Therefore, it is possible that the more severe the PTSD symptomatic is, the more likely it is that a person will develop symptoms of PGD.

As a second goal we examined whether symptoms of PGD proposed by the PG-13 (Prigerson et al., 2008, 2009) form a separable clinical entity distinct from depression, PTSD, and anxiety. The results of principal axis factoring analysis suggest that the a priori selected pool of items loaded on four distinct factors: The symptoms of depression and the cognitive, emotional, and behavioral symptoms of PGD loaded on the first factor (depression factor); the symptoms of anxiety on the second factor (anxiety factor); the symptoms of PTSD on the third factor (PTSD 
factor); and the symptoms of separation distress of PGD on the fourth factor (PGD factor). These results are comparable with findings of a previous pilot study using the newly proposed criteria, which found that PGD may be an appearance of depression rather than a separate nosological entity (Schaal, Elbert, \& Neuner, 2009b). Therefore, our results do not support earlier findings showing that PGD represents a dimension distinct from depression, PTSD, and anxiety (Boelen, \& van den Bout, 2005; Boelen et al., 2003; Chen et al., 1999; Dillen et al., 2009; Pivar \& Field, 2004). The findings are also not in accordance with Boelen and Prigerson (2007), who demonstrated that the newly proposed criteria for PGD indicate the dimension of emotional distress.

Our findings demonstrate the presence of two dimensions of the newly proposed diagnostic criteria for PGD: the symptoms of emotional, cognitive, and behavioral symptoms that loaded on the depression factor and the symptoms of separation distress that strongly loaded on a separate factor. In accordance with Hogan and colleagues (2004), this shows that some of the prolonged grief symptoms are not distinct from bereavement-related depressive symptom clusters. Nevertheless, our analyses provide evidence that there are grief-specific symptoms (e.g., "yearning," "intense pangs of separation distress") that form a distinct configuration from bereavement-related depressive symptoms. The PGD symptom "feeling stunned or shocked" of the Cognitive, Emotional, and Behavioral Symptom subscale also loaded on the PGD factor, indicating that this item may reflect separation distress rather than emotional distress. Separation distress forms a unique dimension that reflects pathological grief reactions. Similarly, Prigerson and coworkers (1999) argued that symptoms of separation distress are the "core of this grief-related disorder" (p. 68). In their study, yearning, which constitutes the key symptom of the Separation Distress subscale, was the item with the highest sensitivity and specificity. Yearning has emerged as an indicator for the relationship to the deceased (Rubin, Malkinson, \& Witztum, 2008). Only modest factor loadings were found for the PG-13 item "confusion about one's role in life or diminished sense of self." This suggests that this item may not be a good indicator of PGD, depression, PTSD, or anxiety. The composition of the items of the PG-13 may need to be reconsidered before inclusion in DSM V may be realized. According to Rubin et al. 
(2008), major challenges in the establishment of a PGD diagnosis include the consideration of the multidimensional complexity of loss reactions, the examination of the complexity of potential ways for adjustment and maladjustment and the risk of overemphasizing a model stressing pathology. Most researchers investigated grief reactions in populations living in industrialized societies. The fact that $8.0 \%$ of our sample met probable diagnostic criteria for PGD (Schaal et al., 2010) shows that PGD also constitutes a mental health problem in African societies, even many years after the grief-related loss. Notably the PG-13 symptom cluster of separation distress presents a grief-specific dimension in our sample of Rwandan genocide survivors. There is a need to extend research to non-Western cultures before definitive conclusions can be drawn.

Limitations of the study include the lack of application of an instrument that would have allowed determination of a full diagnostic status of affective and anxiety disorders. A cut-off score was used as an indicator for caseness. Using the same instruments as in the present study, Ertl and coworkers (2010) demonstrated that mental health assessments in African languages can achieve reliable and valid data but warrant caution in the unevaluated transfer of cutoff scores. Furthermore, our cross-sectional data do not allow determination of causality. In an ongoing study we examine how PGD symptoms respond to treatment of depression and PTSD and vice versa, in order to see the relatedness of the sets of symptoms. Future research must demonstrate the distinctiveness of the newly proposed diagnostic category from other mental conditions before PGD, as it is defined now, can be viewed as a separate disorder. For the Rwandan context it seems that the separation distress scale may produce valuable clinical information not available using current diagnostic tools. PGD could then be viewed as a subset of symptoms aggravating or promoting depression.

\section{References}

Boelen, P. A., \& Prigerson, H. G. (2007). The influence of symptoms of prolonged grief disorder, depression, and anxiety on quality of life among bereaved adults. A prospective study. European Archives of Psychiatry and Clinical Neuroscience, 257, 444452. 
Boelen, P. A., \& van den Bout, J. (2005). Complicated grief, depression, and anxiety as distinct postloss syndromes: A confirmatory factor analysis study. American Journal of Psychiatry, 162, 21752177.

Boelen, P. A., van den Bout, J., \& de Keijser, J. (2003). Traumatic grief as a disorder distinct from bereavement related depression and anxiety: A replication study with bereaved mental health care patients. American Journal of Psychiatry, 160, 13391341.

Bonanno, G. A., Neria, Y., Mancini, A., Coifman, K. G., Litz, B., \& Insel, B. (2007). Is there more to complicated grief than depression and posttraumatic stress disorder? A test of incremental validity. Journal of Abnormal Psychology, 116, 342351.

Chen, J. H., Bierhals, A. J., Prigerson, H. G., Kasl, S. V., Mazure, C. M., \& Jacobs, S. (1999). Gender differences in the effects of bereavement related psycho logical distress in health outcomes. Psychological Medicine, 29, 367380.

Derogatis, L. R., Lipman, R. S., Rickels, K., Uhlenhut, E. H., \& Covi, L. (1974). The Hopkins Symptom Checklist (HSCL): A self report symptom inventory. Behavioral Science, 19, 115.

Dillen, L., Fontaine, J. R. J., \& Verhofstadt Denève, L. (2009). Confirming the distinctiveness of complicated grief from depression and anxiety among adolescents. Death Studies, 33, 437461.

Dyregrov, A., Gupta, L., Gjestad, R., \& Mukanoheli, E. (2000). Trauma exposure and psychological reactions to genocide among Rwandan children. Journal of Traumatic Stress, 13, 321.

Ertl, V., Pfeiffer, A., Saile, R., Schauer, E., Elbert, T., \& Neuner, F. (2010). Vali dation of a mental health assessment in an African conflict population. Psychological Assessment, 22, 318324.

Eth, S., \& Pynoos, R. S. (1994). Children who witness the homicide of a parent. Psychiatry, 57, 287306.

Foa, E. B., \& Tolin, D. F. (2000). Comparison of the PTSD Symptom Scale Interview Version and the Clinician Administered PTSD Scale. Journal of Traumatic Stress, 13, 181191.

Green, B. L. (2000). Traumatic loss: Conceptual and empirical links between trauma and bereavement. Journal of Personal and Interpersonal Loss, 5, 117.

Green, B. L., Krupnick, J. L., Stockton, P., Goodman, L., Corcoran, C., \& Petty, R. (2001). Psychological outcomes associated with traumatic loss in a sample of young women. American Behavioral Scientist, 44, 817837.

Harrison, L., \& Harrington, R. (2001). Adolescents' bereavement experiences. Prevalence, association with depressive symptoms, and use of services. Journal of Adolescence, 24, 159169.

Hogan, N. S., Worden, J. W., \& Schmidt, L. A. (2004). An empirical study of the proposed complicated grief disorder criteria. Omega: The Journal of Death and Dying, 48, 263277.

Horowitz, M. J., Siegel, B., Holen, A., Bonanno, G. A., Milbrath, C., \& Stinson, C. H. (1997). Diagnostic criteria for complicated grief disorder. American Journal of Psychiatry, 154, 904910.

Kersting, A., Kroker, K., Steinhard, J., Lüdorff, K., Wesselmann, U., Ohrmann, P.,... Suslow, T. (2007). Complicated grief after traumatic loss A 14 month 
follow up study. European Archives of Psychiatry and Clinical Neuroscience, 257, 437443.

Kim, K., \& Jacobs, S. (1991). Pathologic grief and its relationship to other psychi atric disorders. Journal of Affective Disorders, 21, 257263.

Lee, B., Kaaya, S. F., Mbwambo, J. K., Smith Fawzi, M. C., \& Leshabari, M. T. (2008). Detecting depressive disorder with the Hopkins Symptom Checklist 25 in Tansania. International Journal of Social Psychiatry, 54, 7.

Lindy, J. D., Green, B. L., Grace, M., \& Titchener, J. (1983). Psychotherapy with survivors of the Beverly Hills Supper Club fire. American Journal of Psychotherapy, 37, 593610.

Melhem, N. M., Day, N., Shear, M. K., Day, R., Reynolds, C. F., \& Brent, D. (2004). Traumatic grief among adolescents exposed to a peer's suicide. American Journal of Psychiatry, 161, 14111416.

Melhem, N. M., Moritz, G., Walker, M., Shear, K., \& Brent, D. (2007). Phenomen ology and correlates of complicated grief in children and adolescents. Journal of the American Academy of Child and Adolescent Psychiatry, 46, 493499.

Mollica, R. F., Wyshak, G., de Marneffe, D., Khuon, F., \& Lavelle, J. (1987). Indochinese versions of the Hopkins Symptom Checklist 25: A screening for the psychiatric care of refugees. American Journal of Psychiatry, 144, 497500.

Momartin, S., Silove, D., Manicavasagar, V., \& Steel, Z. (2004). Complicated grief in Bosnian refugees: Associations with posttraumatic stress disorders and depression. Comprehensive Psychiatry, 45, 475482.

Neria, Y., Gross, R., Litz, B., Maguen, S., Insel, B., Seirmarco, G., \& Marshall, R. D. (2007). Prevalence and psychological correlates of complicated grief among bereaved adults 2.5 3.5 years after September 11th attacks. Journal of Traumatic Stress, 20, 251262.

Neria, Y., \& Litz, B. (2004). Bereavement by traumatic means: The complex syn ergy of trauma and grief. Journal of Loss and Trauma, 9, 7388.

Pasternak, R. E., Reynolds, C. F., Schlernitzauer, M., Hoch, C. C., Buysse, D. J., Houck, P. R., \& Perel, J. M. (1991). Acute open trial nortriptyline therapy of bereavement related depression in late life. Journal of Clinical Psychiatry, 52, 307310 .

Pfefferbaum, B., Call, J. A., Lensgraf, S. J., Miller, P. D., Flynn, B., Doughty, D. E., \& Dickson, W. L. (2001). Traumatic grief in a convenience sample of victims seeking support services after a terrorist incident. Annals of Clinical Psychiatry, 13, 1924.

Pham, P. N., Weinstein, H. M., \& Longman, T. (2004). Trauma and PTSD in Rwanda. Journal of the American Medical Association, 292, 602612.

Pivar, I. L., \& Field, N. P. (2004). Unresolved grief in combat veterans with PTSD. Anxiety Disorders, 18, 745755.

Prigerson, H. G., Bierhals, A. J., Kasl, S. V., Reynolds, C. F., Shear, M. K., Newsom, J. T., \& Jacobs, S. (1996). Complicated grief as a disorder distinct from bereavement related depression and anxiety: A replication study. American Journal of Psychiatry, 153, 14841486.

Prigerson, H. G., Horowitz, M. J., Jacobs, S. C., Parkes, C. M., Aslan, M., Goodkin, K., \& Maciejewski, P. K. (2009). Prolonged grief disorder: 
Psychometric validation of criteria proposed for DSM V and ICD 11. PLoS Medicine, 6(8), 112.

Prigerson, H. G., Shear, M. K., Jacobs, S. C., Reynolds, C. F., Maciejewski, P. K., Davidson, J. R. T., \& Zisook, S. (1999). Consensus criteria for traumatic grief. British Journal of Psychiatry, 174, 6773.

Prigerson, H. G., Vanderwerker, L. C., \& Maciejewski, P. K. (2008). A case for inclusion of prolonged grief disorder in DSM V. In M. S. Stroebe, R. O. Hansson, H. A. W. Schut, W. Stroebe \& E. Van den Blink (Eds.), Handbook of bereavement research and practice: Advances in theory and intervention (pp. 165 186). Washington, DC: American Psychological Association.

Pynoos, R. S., \& Nader, K. (1988). Psychological first aid and treatment approach to children exposed to community violence: Research implications. Journal of Traumatic Stress, 1, 445473.

Reynolds, C. F., Miller, M. D., Pasternak, R. E., Frank, E., Perel, J. M., Cornes, C., ... Kupfer, D. J. (1999). Treatment of bereavement related major depress ive episodes in later life: A controlled study of acute and continuation treat ment with nortriptyline and interpersonal psychotherapy. American Journal of Psychiatry, 156, 202208.

Rubin, S. S., Malkinson, R., \& Witztum, E. (2008). Clinical aspects of a DSM complicated grief diagnosis: Challenges, dilemmas, and opportunities. In M. S. Stroebe, R. O. Hansson, H. A. W. Schut, W. Stroebe, \& E. Van den Blink (Eds.), Handbook of bereavement research and practice: Advances in theory and intervention (pp. 187 206). Washington, DC: American Psychological Association.

Rynearson, E. K., \& McCreery, J. M. (1993). Bereavement after homicide: A synergism of trauma and loss. American Journal of Psychiatry, 150, 258261.

Schaal, S., \& Elbert, T. (2006). Ten years after the genocide: Trauma confron tation and posttraumatic stress in Rwandan adolescents. Journal of Traumatic Stress, 19, 95105.

Schaal, S., Elbert, T., \& Neuner, F. (2009a). Narrative exposure therapy versus group interpersonal psychotherapy: A controlled clinical trial with orphaned survivors of the Rwandan genocide. Psychotherapy and Psychosomatics, 78, 298306.

Schaal, S., Elbert, T., \& Neuner, F. (2009b). Prolonged grief disorder and depression in widows due to the Rwandan genocide. Omega: The Journal of Death and Dying, 59, 203219.

Schaal, S., Jacob, N., Dusingizemungu, J. P., \& Elbert, T. (2010). Rates and risks for prolonged grief disorder in a sample of orphaned and widowed genocide survivors. BMC Psychiatry, 10, 55. doi:10.1186/1471 244X 1055

Schut, H. A. W., De Keijser, J., Van Den Bout, J., \& Dijkhuis, J. H. (1991). Post traumatic stress symptoms in the first years of conjugal bereavement. Anxiety Research, 4, 224234.

Silverman, G. K., Jacobs, S. C., Kasl, S. V., Shear, N. K., Maciejewski, P. K., Noaghiul, F. S., \& Prigerson, H. G. (2000). Quality of life impairments asso ciated with diagnostic criteria for traumatic grief. Psychological Medicine, 30, 857862.

Simon, N. M., Shear, K. M., Thompson, E. H., Zalta, A. K., Perlman, C., Reynolds, C. F., \& Silowash, R. (2007). The prevalence and correlates of 
psychiatric comorbidity in individuals with complicated grief. Comprehensive Psychiatry, 48, 395399.

Vinkers, D. J., Gussekloo, J., Stek, M. L., Westendorp, R. G. J., \& van der Mast, R. C. (2004). The 15 item Geriatric Depression Scale (GDS 15) detects changes in depressive symptoms after a major negative life event: The Leiden 85 plus Study. International Journal of Geriatric Psychiatry, 19, 8084.

Winokur, A., Winokur, D. F., Rickels, K., \& Cox, P. S. (1984). Symptoms of emotional stress in family planning service: Stability over a four week period. British Journal of Psychiatry, 144, 395399. 